\title{
Spectrum of spatial frequency of terahertz vortex Bessel beams formed using phase plates with spiral zones
}

\author{
Zhabin V.N., \\ Novosibirsk State University \\ Volodkin B.O., \\ Samara State Aerospace University \\ Knyazev B.A., \\ Budker Institute of Nuclear Physics \& Novosibirsk State University \\ Mitkov M.S., \\ Budker Institute of Nuclear Physics \\ Pavelyev V.S., \\ Samara State Aerospace University, \\ Image Processing Systems Institute, Russian Academy of Sciences \\ Choporova Yu.Yu. \\ Budker Institute of Nuclear Physics \& Novosibirsk State University
}

\begin{abstract}
This paper presents the first numerical and experimental investigation into the angular spectrum of terahertz Bessel beam with orbital angular momentum generated by a phase plate with spiral zones. The plate was exposed to a Gaussian beam of the Novosibirsk free electron laser. The Bessel beam formed was passed through a collecting lens. The distribution of the intensity of radiation with a wavelength of 141 microns before and after the focusing lens was recorded by a microbolometer array, which was moved along the optical axis by a motorized translation stage. The experimentally measured intensity distributions over the beam cross section recorded along the optical axis are in good agreement with numerical calculations.
\end{abstract}

Keywords: terahertz Bessel beam, free electron laser, phase plate with spiral zones

Citation: Zhabin V.N., Volodkin B.O., Knyazev B.A., Mitkov M.S., Pavelyev V.S., Choporova Yu.Yu. Spectrum of spatial frequency of terahertz vortex Bessel beams formed using phase plates with spiral zones. Proceedings of 
Information Technology and Nanotechnology (ITNT-2015), CEUR Workshop Proceedings, 2015; 1490: 171-178. DOI: 10.18287/1613-0073-2015-1490-171178

\section{Introduction}

A plane electromagnetic wave is characterized by polarization in addition to the amplitude and wave vector. In a general case, an elliptical polarization can be split into the right and left circular polarizations corresponding to spin angular momentum (SAM) of the photon $\pm \hbar$. If the angular dependence of the wave function is $e^{i l \phi}$, the photon has, in addition to the spin, the orbital angular momentum (OAM) $\pm l \hbar$, where $l$ is any integer. Such beams have helical phase fronts with the number of intertwined helices depending on the magnitude of $l$ [1].

In this work we investigated vortex Bessel beams, which are described by the following formula:

$F(r, \phi, z, t)=J_{l}(\kappa r) e^{i l \phi} e^{i k_{z} z} e^{-i \omega t}$

where $J_{l}(x)$ is the Bessel function of the first kind of the $l$ th order, $\kappa$ is the radial component of the wave vector.

In this paper we investigated the angular spectrum of Bessel beam formed by a phase diffractive element [2]. Actually, the spectrum is an expansion of the initial wave into plane waves, which enables better understanding of the structure and behavior of such beams. In particular, one can experimentally check whether a beam is a Bessel one. From the mathematical point of view, the angular spectrum is the Fourier transform. If done with a thin lens, it can be written as follows:

$\frac{k}{f} i^{-l-1} e^{i l \psi} \int_{0}^{R} r J_{l}(\kappa r) J_{l}\left(\frac{k \rho r}{f}\right) d r$.

where $R$ is the radius of the lens aperture; $f$ is the focal distance; $\rho$ and $\psi$ are the polar coordinates in the focal plane. With an infinite lens aperture, the angular distribution takes the form of a thin ring described with the following formula:

$$
\frac{i^{-l-1}}{\kappa} e^{i l \psi} \delta\left(\rho-\rho_{0}\right),
$$

where $\rho_{0}=\frac{\kappa f}{k}$ is the radius of the ring. In case of finite lens aperture, the main ring smears, and there are a number of subordinate concentric rings near it.

\section{Generation of Bessel beams}

There are many ways to produce a vortex beam from a plane wave, e.g. with diffractive elements, which are particularly suitable for the terahertz range. They can 
be forked diffraction gratings or a spiral Fresnel plate. The application of amplitude spiral Fresnel plate to formation of beams with a spatial singularity was proposed in [3]. The boundaries of the zones of the plate are described by the following formula:

$l \varphi=\left(n+\frac{1}{2}\right) \pi+\frac{k r^{2}}{2 f}$,

where $l$ is the topological charge of the beam; $f$ is the focal distance of the plate; $n=0, \pm 1, \pm 2, \ldots$ If $l=0$, it is a conventional Fresnel zone plate.

The experiment was conducted with similar binary phase plates of $30 \mathrm{~mm}$ in diameter produced by reactive ion etching [4] on a high-resistivity silicon wafer but with a constant width of the zones, as shown in Fig. 1. The boundaries of the zones are described by the following formula:

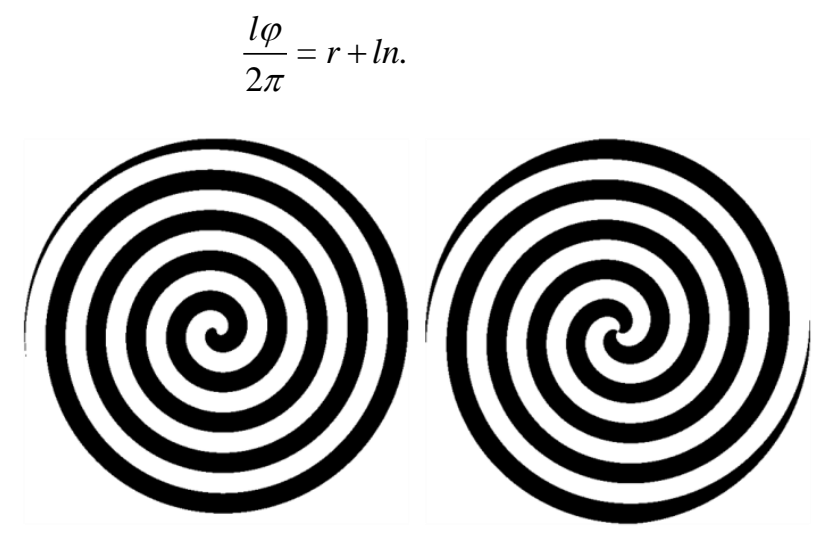

Fig. 1. - Phase plates with spiral zones to form beams with topological charge $l= \pm 1$ (left) and $l= \pm 2$ (right); the charge sign changes when the plate is rotated through an angle of $180^{\circ}$ around the vertical axis. The phase shift between the black and the white areas is $\pi$

Hereinafter this phase plate with spiral zones is referred to as a spiral phase plate (SPP). It was confirmed experimentally (see below) that a plane wave incident on it forms a Bessel beam. If $l=0$, it is a usual axicon.

The numerical modelling was performed in the approximation of the scalar diffraction theory. In the Fresnel approximation the Kirchhoff integral yields an expression of the following form:

$$
E(x, y)=\frac{e^{i k z}}{i \lambda z} \int_{S} E(\xi, \eta) \exp \left[\frac{i k}{2 z}\left((x-\xi)^{2}+(y-\eta)^{2}\right)\right] d \xi d \eta \text {. }
$$

This integral is a two-dimensional convolution of two functions and can be expressed using the Fourier transform, 
$E(x, y)=2 \pi \frac{e^{i k z}}{i \lambda z} \mathrm{~F}_{-}\left\{\mathrm{F}_{+}[E(x, y)] \cdot \mathrm{F}_{+}[w(x, y)]\right\}$,

where $w(x, y)=\exp \left[\frac{i k}{2 z}\left(x^{2}+y^{2}\right)\right]$. This approach can significantly facilitate the numerical simulation of diffraction with the fast Fourier transform.

For numerical calculations it is necessary to turn from the continuous Fourier transform to the discrete Fourier transform (DFT), which can be done in accordance with the following formulas:

$$
\begin{aligned}
& X_{m n}=\sum_{k=0}^{M-1 N-1} \sum_{l=0}^{N l} X^{-\frac{2 \pi i}{M} m k} e^{-\frac{2 \pi i}{N} n l}, \\
& x_{k l}=\frac{1}{M N} \sum_{n=0}^{M-1} \sum_{m=0}^{N-1} X_{m n} e^{\frac{2 \pi i}{M} m k} e^{\frac{2 \pi i}{N} n l} .
\end{aligned}
$$

\section{Modeling and experimental verification}

Computer program Huygens for OS Windows on x86-64 processors was designed for numerical simulation. The library FFTW was used to calculate fast Fourier transform. We carried out the numerical simulation by a scheme corresponding exactly to the experimental set-up shown in Fig. 2. The radiation source was the Novosibirsk free electron laser [5]. In this experiment it was tuned to a wavelength of 141 microns, the phase plates to generate beams with the topological charge were calculated for.

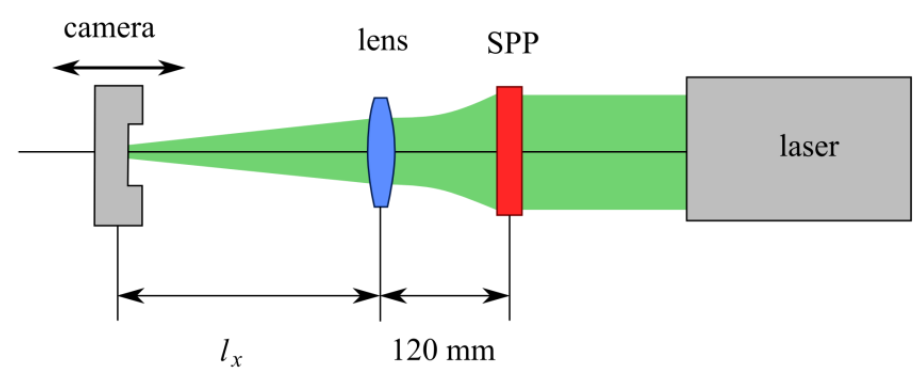

Fig. 2. - Schematic of the experiment. SPP is spiral phase plate

A series of numerical simulations was carried out. In the first numerical simulation, the diffraction of plane wave on a spiral phase plate was investigated, as well as diffraction of ideal Bessel beam on a circular aperture, the radius of which corresponded to the aperture of the plate. From the simulation results the corresponding parameters of the Bessel beam were found. For example, it was found that a plate with the index $l=2$ is best matched by a Bessel beam with the radial 
parameter $\kappa=1.6 \mathrm{~mm}^{-1}$. Fig. 3 presents the respective images. Hereinafter, the brightness corresponds to the intensity and the phase distribution is shown with color.
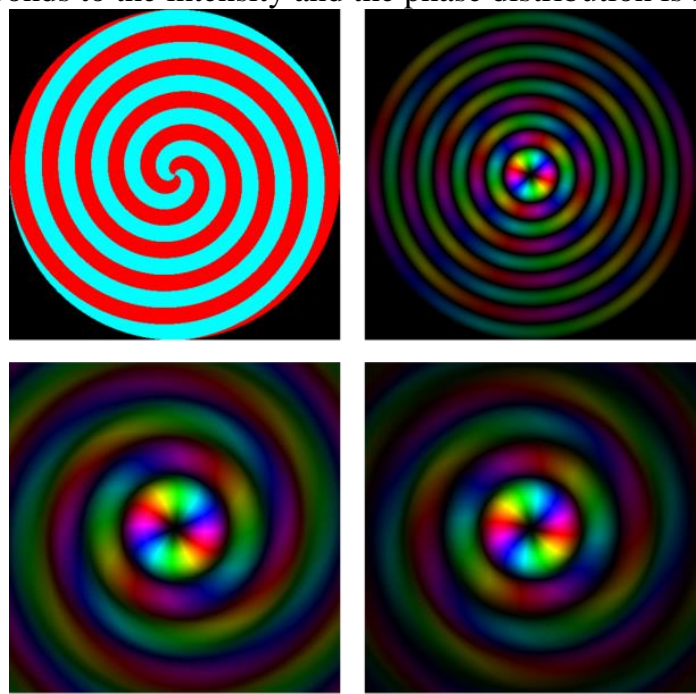

0

Fig. 3. - Comparison of plane wave diffraction on SPP (left) and Bessel beam diffraction on aperture (right)

The second simulation modeled images of ideal Bessel beam of finite radius and plane wave diffracted on a SPP in the Fourier plane of a converging lens with the focal length $f=50 \mathrm{~mm}$, which are shown at Fig. 4. A comparison with theoretical data shows the images in the Fourier plane to be in qualitative agreement with the theoretical predictions, i.e. there is a ring-shape spot. However, there is some difference, i.e. the ring shape is distorted into a fragment of a number of spirals when SPP was used. An analysis of distribution formed by a spiral phase plate is given in $[6,7]$.

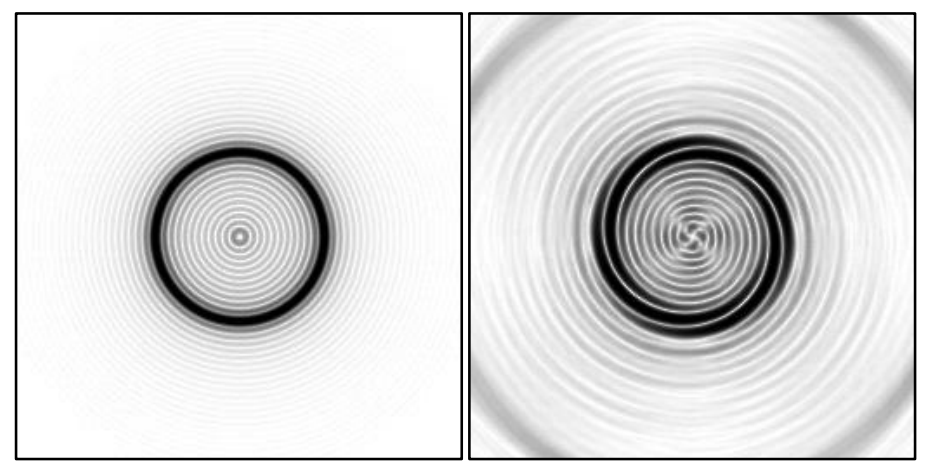

Fig. 4. - Images in Fourier plane of ideal Bessel beam (left), and plane wave diffracted on SPP with $l=2$ (right) 
In the real experiment, the spiral phase plates were exposed to a Gaussian beam of the Novosibirsk free electron laser $I: \exp \left(-2 r^{2} / w^{2}\right)$ with $w=15.1 \mathrm{~mm}$. The radiation intensity distribution along the Bessel beam and in the area behind a TPX lens with a focal length of $50 \mathrm{~mm}$ or $100 \mathrm{~mm}$ was recorded with a microbolometer array (MBA) as a video at 20 frames per second. The MBA was moved along the optical axis by a motorized translation stage $300 \mathrm{~mm}$ long. The size of the sensitive elements (pixel) of the MBA was $51 \times 51 \mu \mathrm{m}^{2}$, which is three times as small as the wavelength of the radiation used. The physical size of the matrix was $16.36 \times 12.24 \mathrm{~mm}^{2}$.

Then the experimental data were compared with the results of the numerical simulation. The calculated and experimentally measured intensity distributions are shown in Fig. 5. It is seen that the experiment and the numerical simulation yield a difference from the ring-shaped image as a spiral fragment. There is a visible substantial difference, the bright spot in the center of the image in the experiment, which indicates that after passing through the SPP some radiation retains the initial direction of the wave vector. As for the rest, the numerical simulation and the experiment are in qualitative agreement, including the observation of spiral fragment instead of ring as predicted by the theory for an ideal Bessel beam.

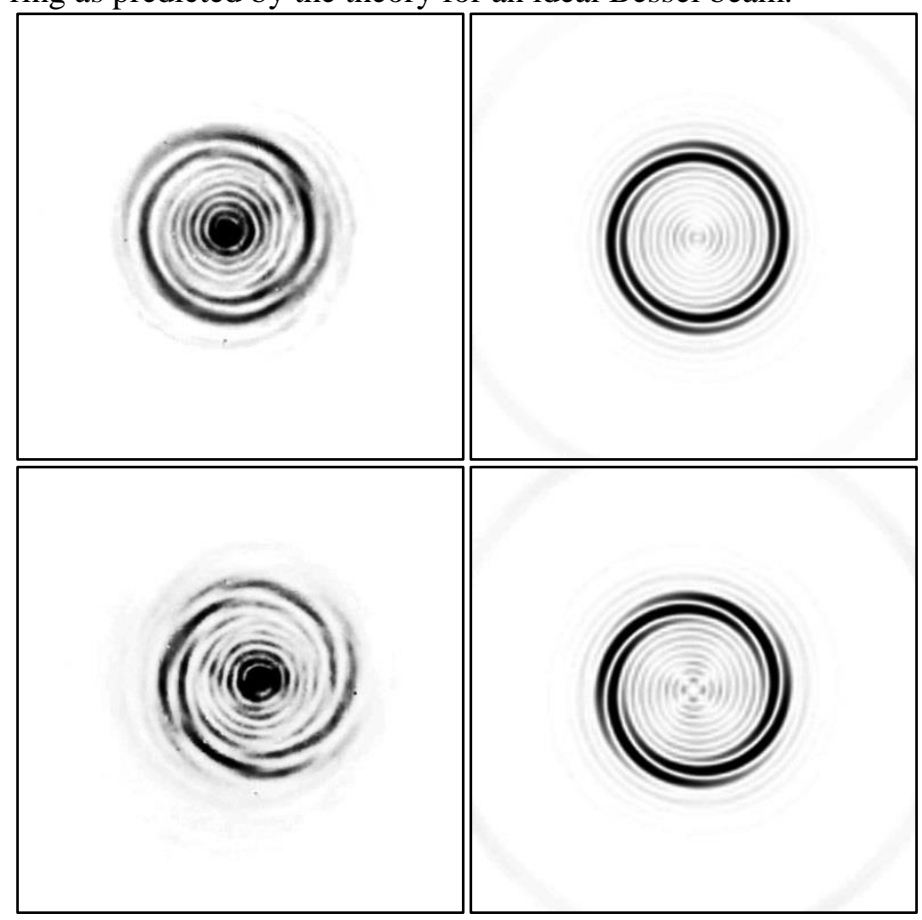

Fig. 5. - Distribution of radiation intensity in lens focal plane (inverted and gamma-corrected). Left: images recorded with MBA. Right: results of numerical modeling for SPP with $l=1$ (top) and $l=2$ (bottom) with lens with $f=50 \mathrm{~mm}$ 
To explain the experimental results, we have carried out additional calculations in which we varied the structure of the plates. The results are presented in Fig. 6. To reveal weak higher-order angular spectrum components, we present images with a low contrast $\gamma=0.15$ ( $\gamma=0.10$ for the amplitude plate). For both amplitude and phase binary plates, spiral-like distorted angular distributions were obtained similar to those shown in Fig. 5. For the plates with linearly growing phase within a zone (kinoform phase plate), the angular distribution is a ring which has to be a perfect Bessel beam. Technically valuable to investigate how the angular distribution changes, if besides the binary plates to use multilevel ones. The last two pictures clearly demonstrate that the three-level and four-level plates generate combination of practically perfect Bessel beam with weak components of higher orders. The latter can be easily removed using a simple system of optical filtering.

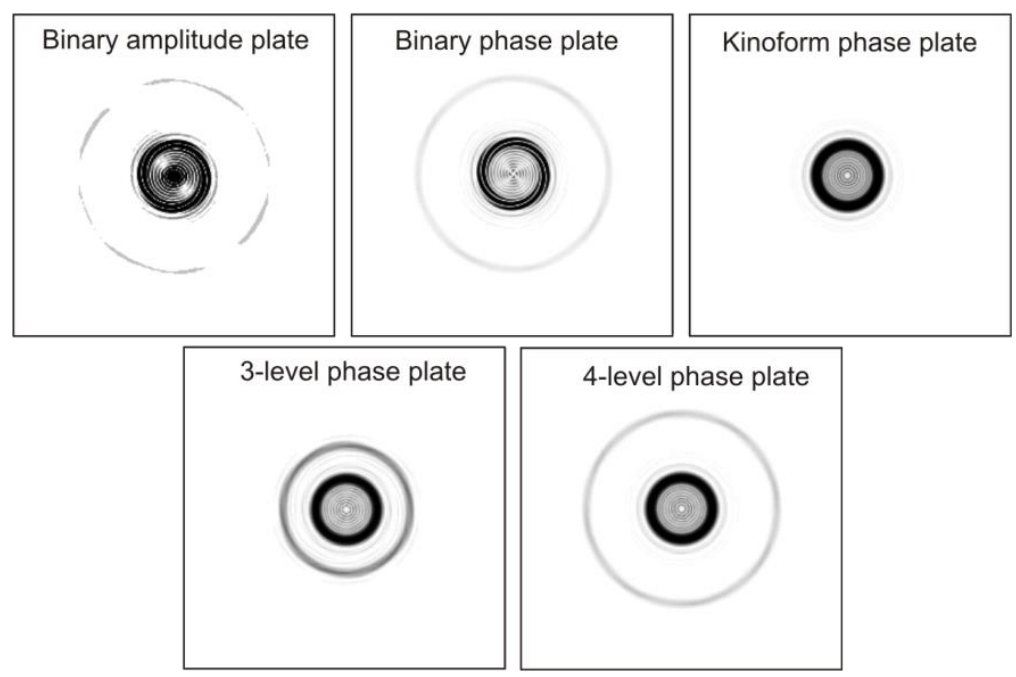

Fig. 6. - Distributions of radiation intensity in the lens focal plane $(f=50 \mathrm{~mm})$ for vortex beams with $l=2$ formed with the spiral plase plates of different structure

\section{Conclusion}

In summary, this study was the very first investigation into the angular distribution of Bessel beam formed by a spiral phase plate in the terahertz frequencies range, which showed good agreement between the results of computer simulation, the theory and the results of the optical experiments. It is shown that perfect Bessel beam can be achieved using multi-level phase spiral plates.

\section{Acknowledgements}

The experimental studies were supported by the Ministry of Education of the Russian Federation and RFBR grant 15-02-06444. The DOEs were developed and fabricated with the support of the Ministry of Education of the Russian Federation, 
including the program for enhancement of the competitiveness of SSAU for years 2013-2020, the State targets to universities and scientific organizations in the field of scientific activity, project 1879 and RFBR grant 13-02-97007. The equipment for investigating the characteristics of the DOEs was made with the support of Russian Science Foundation grant 14-50-00080. The experiments were performed using the equipment of the shared-equipment center "Siberian Center for Synchrotron and Terahertz Radiation (SEC SCSTR)."

\section{References}

1. Twisted Photons. Applications of Light with Orbital Angular Momentum. Willey, 2011.

2. Knyazev BA, Choporova YuYu, Pavelyev VS, Volodkin BO, Mitkov MS. High-Power Terahertz Non-Diffractive Bessel Beams with Angular Orbital Momentum. Generation and Application. Sourse: 〈http://www.irmmw-thz2015.org/paper 3129943>.

3. Heckenberg RN, McDuff R, Smith CP, White AG. Generation of optical phase singularities by computer-generated holograms. Optics Letters, 1992; 17: 221-223,

4. Agafonov AN, Volodkin BO, Kaveev AK, Kachalov DG, Knyazev BA, Kropotov GI, Tukmakov KN, Pavelyev VS, Tsypishka DI, Choporova YuYu. Binary DOE with elongated focal depth to focus terahertz free electron laser radiation (NOVOFEL). Computer Optics, 2015; 39(1): 58-63. [in Russian]

5. Kulipanov GN, Bagryanskaya EG, Chesnokov EN, Choporova YuYu, Gerasimov VV, Getmanov YaV, Knyazev BA, Kubarev VV, Peltek SE, Popik VM, Salikova TV, Scheglov MA, Seredniakov SS, Shevchenko OA, Skrinsky AN, Veber SL, Vinokurov NA. Novosibirsk free electron laser: Facility description and recent experiments. IEEE Transactions on Terahertz Science and Technology, 2015; 5(5): in print.

6. Soifer VA. Diffractive computer-based optics. Moscow: FIZMATLIT, 2007. 736 p. [in Russian]

7. Gavrilov AV, Golovashkin DL, Doskolovich LL, Dyachenko PN, Khonina SN, Kotlyar VV, Kovalev AA, Nalimov AG, Nesterenko DV, Pavelyev VS, Shuyupova YO, Skidanov RV, Soifer VA. In Diffracrive Nanophotonics. Edited by V.A. Soifer. CRC Press. Taylor\&Francis Group. CISP. Boca Raton, 2014. 679 p. 